\title{
Acceleration: problems and progress
}

\author{
Donald B. Melrose \\ School of Physics, University of Sydney, NSW 2006, Australia \\ email: melrose@physics.usyd.edu.au
}

\begin{abstract}
Older ideas on acceleration are linked to current ideas to identify both the major successes in the field, and long-standing problems and difficulties that remain unresolved.
\end{abstract}

Keywords. acceleration of particles, shock waves, plasmas

\section{Introduction}

The recent literature on acceleration separates into four broad applications: to space plasmas, for which we have in situ data; to solar particles, for which we have both signatures of the particles in the solar atmosphere and direct measurements of particles that escape: to Galactic cosmic rays (CR); and to synchrotron sources, AGN, bursters, etc., for which we have only the electromagnetic spectrum. Rather than attempt to review progress in all these areas, I take a look back to early ideas on acceleration, consider the progress we have made, and the major problems that have remained unsolved for decades.

\section{Brief historical review}

Swann (1933) suggested that solar CR are accelerated by a changing magnetic field (betratron effect), and he estimated the available potential to be $\Phi \approx 10^{10} \mathrm{~V}$. Although Swann's specific model is no longer taken seriously, there are two important ideas that remain relevant today: effective acceleration is due to inductive electric fields, and the maximum potential available can be estimated from simple arguments, e.g., $\Phi \approx B L v$ for magnetically connected conducting regions separated by $L$ in relative motion at speed $v$ or, for a unipolar inductor, $\Phi \approx\left(Z_{0} P\right)^{1 / 2}$, where $Z_{0} \simeq 300 \Omega$ is the impedance of free space and $P$ is the power. Fermi (1949) suggested that $\mathrm{CR}$ are accelerated by reflections off moving interstellar clouds, and this became the prototype for stochastic acceleration. It is now recognized that the acceleration corresponds to an isotropic diffusion of particles in momentum space (Tverskoi 1967) that can be attributed to the damping of compressive MHD waves (Achterberg 1981). Fermi (1954) proposed first-order acceleration between 'closing jaws' and this idea underpins diffusive shock acceleration (DSA), proposed independently by various authors in 1997-8 (e.g., the review by Malkov \& Drury 2001) - a major success in this field. An essential ingredient in both stochastic acceleration and DSA is the efficient scattering of fast particles. Another major success was the recognition in the mid 1950s that resonant scattering by waves causes pitch-angle diffusion and spatial diffusion along field lines; moreover, anisotropic particles cause their resonant waves to grow (e.g., Melrose 1980), but this is ineffective at high energy.

\section{Outstanding problems}

In simple models for DSA, effective diffusion at high energy and across field lines are simply postulated, without adequate justification. Ideas on how these problems might be addressed were suggested by Bell (2004) and Achterberg \& Ball (1994), respectively. 
The Galactic Cosmic Ray (GCR) CR spectrum is separated into (Axford 1994) GCRI at $<10^{15} \mathrm{eV}$, GCRII and EGCR. GCRI is attributed to DSA by shocks from SN explosions that permeate the ISM. GCRI has nearly normal cosmic abundance implying that the injection into DSA is insensitive to charge $(Z)$ and mass $(A)$ - maybe super-Alfvénic jets of fluid due to magnetic reconnection. This contrasts with anomalous abundances in other contexts, notably overabundance of ${ }^{3} \mathrm{He}$, notably in 3 Cent. A (Sargent \& Jugaku 1961) and in some solar particle events. Sensitivity to $Z, A$ in pre-acceleration by cyclotron waves (Fisk 1978) remains the favored explanation (Liu, Petrosian \& Mason 2006). The acceleration of the GCRII and its relation to GCRI is still a topic of debate, and the acceleration of the presumably extragalactic EGCR is a major unsolved problem.

Flat synchrotron spectra correspond to $f(p) \propto p^{-3}$, while DSA at a single nonrelativistic shock implies $f(p) \propto p^{-b}$ with $b>4$. The 'cosmic conspiracy' model for flat spectra involves broadening a self-absorbed peak due to a special geometry (Cotton et al. 1980). Flat spectra can be produced by DSA at multiple shocks (White 1985): energy gains, due to DSA at each shock, and losses, due to decompression between shocks, constitute a stochastic acceleration mechanism. Synchrotron pile-up occurs for $b<4$, and DSA at multiple shocks with synchrotron losses is an alternative model for flat spectra (Melrose \& Crouch 1997). The emission is dominated by the compressed regions around the shocks.

A major outstanding problem is 'bulk energization' of electrons to $10-100 \mathrm{keV}$ in solar flares. Magnetic energy is favored, but this must involve a statistically large number of tiny dissipation regions. How these are coupled together and how all the electrons can be processed through a tiny net volume is unclear. Network theory (e.g., Vlahos, Isliker \& Lepreti 2004) might provide a useful framework.

\section{Conclusions}

Progress has been slow, with resonant scattering, stochastic acceleration and DSA being major successes. Many problems remain inadequately understood, and many more could be added to my list.

\section{References}

Achterberg, A. 1981, A\& A, 97, 259

Achterberg, A., \& Ball, L. $1994 A \& A, 285,687$

Axford, W.I. 1994, ApJS, 90, 937

Bell, A.R. 2004, MNRAS, 353, 550

Cotton, W.D., Wittels, J.J., Shapiro, I.I., et al. 1980, ApJ, 238, L123

Fermi, E. 1949, Phys. Rev., 75, 1169

Fermi, E. 1954, ApJ, 119, 1

Fisk, L.A. 1978, ApJ, 224, 1048

Liu, S., Petrosian, V., \& Mason, G.M. 2006, ApJ, 636, 462

Malkov, M.A., \& Drury, L.O.'C. 2001, Rep. Prog. Phys., 64, 429

Melrose, D.B. 1980, Plasma Astrophysics II (New York: Gordon \& Breach), p. 12

Melrose, D., \& Crouch, A. 1997, Pub. Astron. Soc. Aust., 14, 251

Sargent, W.L.W., \& Jugaku, J. 1961, ApJ, 134, 777

Swann, W.F.G. 1933, Phys. Rev., 43, 217

Tverskoi, B.A. 1967, Sov. Phys. JETP, 25, 317

Vlahos, L., Isliker, H., \& Lepreti, F. 2004, ApJ, 608, 540

White, R.L. 1985, ApJ, 289, 698 